\title{
Structural basis for EGFR ligand sequestration by Argos
}

\author{
Daryl E. Klein, Steven E. Stayrook, Fumin Shi, Kartik Narayan, and Mark A. Lemmon \\ Department of Biochemistry and Biophysics, University of Pennsylvania School of Medicine, $809 \mathrm{C}$ \\ Stellar-Chance Laboratories, 422 Curie Boulevard, Philadelphia, PA 19104-6059, U.S.A.
}

\section{Abstract}

Members of the Epidermal Growth Factor Receptor (EGFR) or ErbB/HER family and their activating ligands are essential regulators of diverse developmental processes ${ }^{1,2}$. Inappropriate activation of these receptors is a key feature of many human cancers ${ }^{3}$, and its reversal is an important clinical goal. A natural secreted antagonist of EGFR signalling, called Argos, was identified in Drosophila ${ }^{4}$. We showed previously that Argos functions by directly binding (and sequestering) growth factor ligands that activate $\mathrm{EGFR}^{5}$. Here we describe the $1.6 \AA$ A resolution crystal structure of Argos bound to an EGFR ligand. Contrary to expectations ${ }^{4,6}$, Argos contains no EGF-like domain. Instead, a trio of closely-related domains (which resemble a three-finger toxin fold ${ }^{7}$ ) form a clamplike structure around the bound EGF ligand. Although structurally unrelated to the receptor, Argos mimics EGFR by using a bipartite binding surface to entrap EGF. The individual Argos domains share unexpected structural similarities with the extracellular ligand-binding regions of TGF $\beta$ family receptors 8 . The three-domain clamp of Argos also resembles the urokinase-type plasminogen activator (uPA) receptor, which uses a similar mechanism to engulf the uPA EGF-like module ${ }^{9}$. Our results suggest that undiscovered mammalian counterparts of Argos may exist among other poorly characterized structural homologues. In addition, the structures presented here define requirements for the design of artificial EGF-sequestering proteins that would be valuable anti-cancer therapeutics.

The 419-residue mature D. melanogaster Argos was modified to improve crystallization by removing a poorly conserved region with multiple O-linked glycosylation sites (residues 1-87). In addition, a non-conserved 120-amino acid insert (residues 140-259) exclusive to drosophilid Argos molecules was replaced with the corresponding 5 amino acids (PDGRT) from Apis mellifera Argos (Supplementary Fig. 1). The resulting 217-amino acid protein (Argos 217 ) exhibits greatly improved stability and purification properties, and binds Spitz with an affinity $\left(\mathrm{K}_{\mathrm{D}}=7.7 \mathrm{nM}\right.$ : Supplementary Fig. 2$)$ similar to that previously measured ${ }^{5}$ for full-length $\operatorname{Argos}_{419}\left(K_{\mathrm{D}}=20 \mathrm{nM}\right)$. Triclinic crystals of Argos 217 bound to the Spitz EGF-domain (Spitz $\mathrm{EGF}$ : residues 48-99) grew at neutral $\mathrm{pH}$ with two complexes per asymmetric unit, and diffracted to $1.6 \AA ̊$ resolution. The structure was solved by multiwavelength anomalous dispersion (MAD), using the halide soak method ${ }^{10}$ (Supplementary Table 1). Representative electron density is shown in Fig. 1a,b. Structures of uncomplexed Argos 217 and Spitz EGF $_{\text {were }}$ also determined (to $2.5 \AA$ and $1.5 \AA$ respectively) by molecular replacement.

Correspondence and requests for materials should be addressed to M.A.L. (e-mail: mlemmon@mail.med.upenn.edu). Reprints and permissions information is available at www.nature.com/reprints.

Author Contributions: D.E.K. and M.A.L. conceived and designed the project. D.E.K. was responsible for all construct design and execution of protein biochemistry, crystallization, and data collection. D.E.K. solved and refined the Argos217/SpitzEGF complex structure. S.E.S. solved and refined the structures of uncomplexed Argos217 and SpitzEGF by molecular replacement using datasets collected by D.E.K. K.N. helped with crystal manipulation and data collection. F.S. performed binding studies with Argos and Spitz variants, as well as analytical ultracentrifugation, directed by D.E.K. D.E.K. and M.A.L. interpreted data and wrote the manuscript.

Coordinates have been deposited in the Protein Data Bank under codes 3CA7 (SpitzEGF), 3C9A (Argos217/SpitzEGF complex), and 3CGU (Argos217 alone).

The authors declare that they have no competing financial interests. 
Argos consists of three separate disulphide-bonded $\beta$-sheet domains (domains 1-3) that show no resemblance to EGF domains. This three-domain composition was not discerned in sequence analyses. The three domains of Argos engulf the bound Spitz ${ }_{\mathrm{EGF}}$ molecule with a structure that is reminiscent of a C-clamp (Fig. 1c,d). Domains 2 and 3 constitute the 'jaws' of this clamp, and make an intimate set of direct contacts with bound SpitzEGF. Domain 1 forms the backbone of the C-clamp and does not contribute directly to ligand binding. Spitz $\mathrm{EGF}$ itself is very similar in structure to other known EGFR family ligands (Supplementary Fig. 3). Its three disulphide bonds generate three loops in the typical EGF domain structure, which are termed the A-, B-, and C-loops (Fig. 1e). The Spitz B-loop protrudes into the crevice between domains 2 and 3 of Argos (Fig. 1c,d). The conformation of Spitz $\mathrm{EGF}_{\text {is }}$ iargely unaltered upon binding to Argos, apart from small changes in the backbone at the B-loop tip (Supplementary Fig. 3) and reorientation of certain interfacial side-chains.

Domains 1, 2 and 3 of Argos superimpose remarkably well (with $\mathrm{C}_{\alpha}$ r.m.s. deviations of

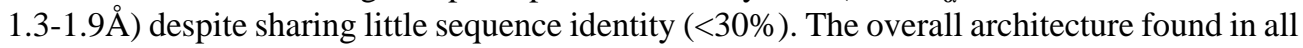
3 domains is shown in Fig. 2a. The cysteines form a disulphide-bonded core, from which two $\beta$-hairpins project to form a four-stranded $\beta$-sheet with a relatively unusual antiparallel 2143 topology 11 . The result is a flat domain that resembles part of a left hand, with the $\beta$-hairpins as two fingers plus a thumb-like projection emerging from the disulphide-bonded core. In domains 1 and 3 (but not domain 2), a knuckle-like protrusion also projects below the plane of the page in Fig. 2a. The positions of the $\mathrm{C} 1-\mathrm{C} 3$ and $\mathrm{C} 4-\mathrm{C} 6$ disulphides are almost identical in all three Argos domains (Fig. 2b), but the third (C2-C5) disulphide is missing from domain 2. The absence of this disulphide correlates with the lack of a knuckle in domain 2. Domain 1 is distinguished by the presence of a unique additional $\mathrm{N}$-terminal $\beta$-strand $\left(\beta 1^{\prime}\right)$ that is parallel to strand $\beta 2$. The drosophilid-specific insertion in Argos occurs at the top of domain 1 in the orientation shown in Fig. 2, immediately before the knuckle (between C4 and C5). This insertion would likely project out and away from domain 1 , with its ends constrained by the C2-C5 and C4-C6 disulphides.

Protein Data Bank searches with the DALI server ${ }^{12}$ and a secondary structure matching (SSM) algorithm ${ }^{13}$ revealed that the three domains of Argos are significantly related to the threefinger toxin fold found in snake neurotoxins and cardiotoxins ${ }^{7}$ - although the disulphidebonding pattern is altered, and Argos has just two (rather than three) fingers per domain. Interestingly, the three-finger toxin fold is also found in the extracellular ligand-binding domains of receptors for TGF $\beta$ family ligands ${ }^{8}$. As shown in Fig. $2 c$, two fingers of the extracellular ligand-binding domain of the type II activin receptor (ActRII) ${ }^{14}$ overlay very well with domain 3 of Argos. Members of the Ly-6 superfamily also share this fold ${ }^{7}$, including the receptor for urokinase-type plasminogen activator (uPA $)^{9}$.

Argos 'clamps' SpitzeGF between domains 2 and 3 (Fig. 1c,d), and buries 35\% of the ligand's surface. Domains 2 and 3 are approximately parallel to one another (Fig. 1c), and are stacked so that they present opposite surfaces to the Spitz $\mathrm{EGF}_{\mathrm{F}}$ molecule sandwiched between them. Using the hand analogy introduced above, domain 3 presents its palm to the bound ligand, whereas domain 2 contacts Spitz $\mathrm{EGF}$ using the back of the hand. The Spitz-binding regions on domains 2 and 3 can each be divided into two sites (Fig. 3): an A-site and a B-site. The A sites $(\mathrm{d} 2 \mathrm{~A}, \mathrm{~d} 3 \mathrm{~A})$ lie on the $\beta$-sheet surfaces, and the $\mathrm{B}$ sites $(\mathrm{d} 2 \mathrm{~B}, \mathrm{~d} 3 \mathrm{~B})$ involve the thumb and disulphide-bonded core. Site A on domain 2 (d2A) consists of a patch of hydrophobic sidechains on the back-of-hand surface of fingers 1 and 2 (L301, L326, Y341, F343), which makes van der Waal's contacts with three aliphatic side-chains from the Spitz B-loop (V72, I74 and V79). Site B on domain 2 (d2B) comprises a flat surface (cyan in Fig. 3) formed by side-chains from the base of finger 2 (V323, Y325, S346 and P347) and the domain 2 thumb (F294). This flat surface packs against the C-terminal part of Spitz $\mathrm{EGF}$, contacting M89, Q91, and Y95 in the C-loop. Importantly, we previously identified S346 and P347 from site d2B as important 
residues in a genetic screen for modifiers of an Argos misexpression phenotype in Drosophila eye development ${ }^{15}$. S346 and P347 are both conserved in all known Argos orthologs

(Supplementary Fig. 1), and make direct hydrogen bonds with Spitz (Fig. 3). In domain 3, part of site A (d3A) involves polar side-chains on the palm side of finger 1 (T363, R365, E373,

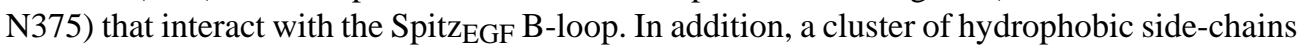
around the tip of finger 2 (F403, L404, I408) contacts residues from the Spitz $\mathrm{EGF}_{\mathrm{N}} \mathrm{N}$-terminus, A-loop, and B-loop. A key feature of this interaction is the projection of the F403 and L404 side-chains into a hydrophobic pocket on the Spitz surface formed by Y52, P55, P78, and Y80 (Fig. 3). In the B-site on domain 3 (d3B), side-chains close to the base of the two fingers (Q357, P358, L361, N377, and S412) form a binding site for the Spitz A-loop helix (contacting Spitz T57, F58, W61, and Y62). Site d3B also accommodates the side-chains of Spitz R92 and L64 (Fig. 3).

Despite having a completely different structural scaffold from EGFR, Argos mimics the characteristic bipartite capture of growth factor seen in ligand-bound structures of the EGFR extracellular region (sEGFR) ${ }^{16,17}$. Argos presents two ligand-binding surfaces that closely resemble those in EGFR. Specifically, domain 2 of Argos mimics domain I of sEGFR in its ligand contacts, whereas domain 3 of Argos emulates sEGFR domain III (Fig. 4a). The primary ligand contacts made by domain 2 of Argos and domain I of EGFR are remarkably similar. Both utilize a central hydrophobic patch that interacts with a similar region of the B-loop of the bound EGF domain. As shown in Fig. 4a (upper panels), Argos site d2A and EGFR site 1 (from Ogiso et al. ${ }^{17}$ ) are very similar - with a comparable arrangement of hydrophobic sidechains making ligand contacts in each case. The second binding site on Argos domain 2 (d2B) also recapitulates many other sEGFR domain $\mathrm{I} / \mathrm{hEGF}$ contacts, but is different in detail. Along the same lines, site B on Argos domain 3 (d3B) recapitulates site 2 in the sEGFR/hEGF interface ${ }^{17}$ (Fig. 4a, lower panels), including interactions with an arginine that is critical for hEGF binding to its receptor (R41 in hEGF, R92 in Spitz). Both sites accommodate three key ligand side-chains (Y13, L15 and R41 in hEGFR: Y62, L64 and R92 in Spitz) in analogous binding sites. Site 3 on domain III of EGFR is not mimicked by Argos; the C-terminus of the

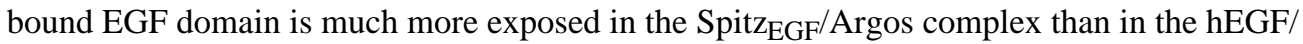
sEGFR complex (Fig. 4a). Argos compensates for the absence of these site 3 interactions with an extensive set of unique contacts mediated by site d3A (Fig. 4a).

Overall, Argos domain 2 buries slightly less surface on $\operatorname{Spitz}_{\mathrm{EGF}}$ (560Å2) than EGFR domain I buries on hEGF $(745 \AA 2){ }^{17}$, but includes more apolar surface (68\%) than in the EGFR domain/ hEGF interface (56\%). Argos domain 3 buries slightly more of its bound EGF domain

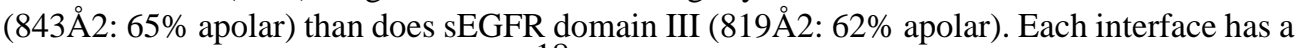
shape complementarity parameter $\left(S_{c}\right)^{18}$ of 0.70 , which is typical for strong protein/protein interfaces and reflects a significantly greater shape complementarity than seen in antibody/ antigen interfaces. The fact that Spitz $\mathrm{EGF}$ binds $\sim 10$-fold more strongly to Argos than to the Drosophila EGFR extracellular region may reflect different requirements for domain rearrangements in the two binding proteins. Clearly defined sets of (intramolecular) domain 1/2 and domain 1/3 contacts (Supplementary Fig. 4) may optimize bipartite capture of Spitz

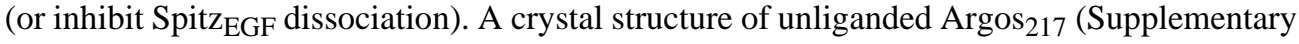
Figs. 5 \& 6) suggests that domain 3 may be mobile, possibly 'collapsing' onto domain 2 in the absence of bound ligand (see Supplementary Information for discussion). Once Spitz $\mathrm{EGF}_{\text {is }}$ bound, domain $1 / 3$ interactions in the complex may slow down its dissociation. Interestingly,

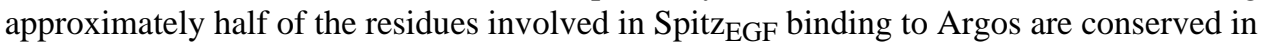
human EGF and/or TGF $\alpha$, which may explain the ability of Argos to bind detectably (although weakly, with $\mathrm{K}_{\mathrm{D}} \sim 5 \mu \mathrm{M}$ ) to human $\mathrm{EGF}$ (not shown).

Sequence analyses have failed to identify clear homologues of Argos in vertebrates, but this does not necessarily mean that functional mammalian analogues do not exist. The amino acid 
sequence of Argos has been unusually cryptic, providing few (or misleading) clues about the structure of the protein. It only became apparent that Argos has three distinct domains (and no EGF-like domain) once the structure described here was determined. Moreover, the relationship of the constituent domains in Argos to the three-finger toxin fold can only been seen in structural (and not sequence) comparisons. As shown in Fig. 2c, the individual domains of Argos share unexpected and striking structural similarity with the extracellular ligandbinding regions of receptors for TGF $\beta /$ bone morphogenetic protein (BMP) family ligands, which consist of little more than a single three-finger toxin fold ${ }^{8,14}$. As shown in Supplementary Fig. 7, the positions of the ligand-binding sites in Argos domain 3 and the extracellular regions of TGF $\beta / B M P$ family receptors also correspond strikingly well. Both utilize the palm side of the domain according to the analogy drawn in Fig. 2a.

Among human proteins that contain three-finger toxin fold domains, one intriguing example uses three such modules to engulf an EGF-like domain. This is the cell-surface receptor for urokinase plasminogen activator (uPA) ${ }^{9,19}$, which represents an unexpected structural homologue of Argos. As shown in Fig. 4b, the three domains from the uPA receptor (uPAR) form a clamp-like structure around the EGF domain found at the amino terminus of uPA. Each domain presents its palm side to the bound ligand (like Argos domain 3). The uPAR structure resembles the clamp formed around the Spitz EGF domain by Argos (Fig. 1c,d). There are differences in the orientation of the bound EGF domain in the Argos/Spitz and uPAR/uPA complexes. Moreover, Argos has one of its three constituent domains (domain 2) 'inverted' so that it presents the back (rather than palm) of the hand to the ligand. However, the correspondence in overall architecture and function (as proteins that entrap EGF domains) of uPAR and Argos suggest that other structural homologues of Argos should be sought in mammals. There are many human uPAR/Ly6 domain-containing proteins for which the function remains unclear. Several, such as CD177/PRV-1 and C4.4A, contain multiple threefinger domains ${ }^{20,21}$ like uPAR. Moreover, C4.4A expression is known to be altered in several metastatic human cancers ${ }^{22}$. We suggest that one of these numerous structural homologues might represent a functional analogue of Argos. Even if such an analogue does not exist, the known human proteins from this class could clearly be used as structural scaffolds in the design of protein therapeutics that will sequester ErbB receptor-activating EGF domains.

It is increasingly clear that excessive or unregulated expression (or shedding) of ErbB family ligands is important in numerous cancers, through autocrine and/or paracrine activation of cell growth $23-27$. The role of ErbB ligands may be particularly important in cancers where available receptor-targeted approaches have failed or have met resistance ${ }^{26}$. In these (and other) cases, therapeutic agents that neutralize ErbB receptor ligands are likely to have great value. The understanding of Spitz neutralization by Argos that we present here provides new avenues to explore in efforts to identify a human homologue of Argos. The structural lessons also provide clear suggestions for which human proteins might be used as scaffolds for generating new protein therapeutics that sequester aberrantly-produced EGF-like growth factors - exploiting a mechanism for inhibiting EGFR signalling that has evolved naturally.

\section{Methods Summary}

\section{Protein purification and crystallization}

$\operatorname{Argos}_{217}$ was produced in baculovirus-infected Spodoptera frugiperda Sf9 cells, using the amino-terminal BiP signal sequence to direct secretion of the protein into the medium. The protein has a hexahistidine tag at its $\mathrm{C}$-terminus, which was used for purification as described $^{5}$. The EGF domain of Spitz (residues 48-99: Spitz EGF) was generated by proteolytic cleavage of a modified form of secreted full-length secreted Spitz produced in transfected Drosophila $\mathrm{S} 2$ cells. Crystals were grown using the hanging drop method. Crystals grew from a 1:1 Argos $217:$ Spitz $E G F$ mixture (250 $\mu \mathrm{M}$ complex) at pH 7, using PEG20000 as the precipitant, 
or from a $250 \mu \mathrm{M}$ solution of $\operatorname{Argos}_{217}$ alone using PEG3350 as precipitant (at $\mathrm{pH} 4.5$ ). Crystals of Spitz $\mathrm{EGF}_{\text {alone }}(500 \mu \mathrm{M})$ grew in ammonium sulphate, $\mathrm{pH}$ 6.5.

\section{Structure determination}

The Argos $217:$ Spitz $_{\mathrm{EGF}}$ structure was determined by multiwavelength anomalous dispersion (MAD) using the halide soak method ${ }^{10}$. Crystallographic data were collected at the Advanced Photon Source (Argonne, IL) and the Advanced Light Source (Berkeley, CA), as summarized in Supplementary Table 1. Phasing from 10 ordered bromine ions yielded a readily interpretable electron density map allowing nearly the entire chain of each complex to be traced. Alternating cycles of model building with COOT $^{28}$ and refinement with REFMAC ${ }^{29}$ led to a complete model of Argos and Spitz with $R_{\text {cryst }}$ and $R_{\text {free }}$ values of 0.20 and 0.24 respectively to $1.6 \AA$ resolution (Supplementary Table 1). The unliganded Argos structure was solved by sequential molecular replacement using PHASER in the CCP4 suite of programs ${ }^{29}$, and the SpitzEGF structure was solved by molecular replacement using a loop-truncated version of the human EGF domain structure (1JL9) ${ }^{30}$.

\section{Supplementary Material}

Refer to Web version on PubMed Central for supplementary material.

\section{Acknowledgements}

We thank members of the Lemmon and Ferguson laboratories, Greg Van Duyne, and Jim Shorter for advice and critical reading of the manuscript. Supported by grants from the NIH (to M.A.L.) and U.S. Army Breast Cancer Research Program (to D.E.K. and M.A.L.).

\section{References}

1. Holbro T, Hynes NE. ErbB receptors: directing key signaling networks throughout life. Annu Rev Pharmacol Toxicol 2004;44:195-221. [PubMed: 14744244]

2. Shilo BZ. Regulating the dynamics of EGF receptor signaling in space and time. Development 2005;132:4017-4027. [PubMed: 16123311]

3. Hynes NE, Lane HA. ERBB receptors and cancer: the complexity of targeted inhibitors. Nature Rev Cancer 2005;5:341-354. [PubMed: 15864276]

4. Freeman M, Klambt C, Goodman CS, Rubin GM. The argos gene encodes a diffusible factor that regulates cell fate decisions in the Drosophila eye. Cell 1992;69:963-975. [PubMed: 1606617]

5. Klein DE, Nappi VM, Reeves GT, Shvartsman SY, Lemmon MA. Argos inhibits epidermal growth factor receptor signalling by ligand sequestration. Nature 2004;430:1040-1044. [PubMed: 15329724]

6. Kretzschmar D, et al. Giant lens, a gene involved in cell determination and axon guidance in the visual system of Drosophila melanogaster. EMBO J 1992;11:2531-2539. [PubMed: 1628618]

7. Tsetlin V. Snake venom $\alpha$-neurotoxins and other 'three-finger' proteins. Eur J Biochem 1999;264:281286. [PubMed: 10491072]

8. Greenwald J, Fischer WH, Vale WW, Choe S. Three-finger toxin fold for the extracellular ligandbinding domain of the type II activin receptor serine kinase. Nat Struct Biol 1999;6:18-22. [PubMed: 9886286]

9. Barinka C, et al. Structural basis of interaction between urokinase-type plasminogen activator and its receptor. J Mol Biol 2006;363:482-495. [PubMed: 16979660]

10. Dauter Z, Dauter M, Rajashankar KR. Novel approach to phasing proteins: derivatization by short cryo-soaking with halides. Acta Crystallogr D Biol Crystallogr 2000;56(Pt 2):232-237. [PubMed: 10666615]

11. Zhang C, Kim SH. The anatomy of protein $\beta$-sheet topology. J Mol Biol 2000;299:1075-1089. [PubMed: 10843859] 
12. Holm L, Sander C. Protein structure comparison by alignment of distance matrices. J Mol Biol 1993;233:123-138. [PubMed: 8377180]

13. Krissinel E, Henrick K. Secondary-structure matching (SSM), a new tool for fast protein structure alignment in three dimensions. Acta Crystallogr D Biol Crystallogr 2004;60:2256-2268. [PubMed: 15572779]

14. Allendorph GP, Vale WW, Choe S. Structure of the ternary signaling complex of a TGF-beta superfamily member. Proc Natl Acad Sci U S A 2006;103:7643-7648. [PubMed: 16672363]

15. Alvarado D, Evans TA, Sharma R, Lemmon MA, Duffy JB. Argos mutants define an affinity threshold for spitz inhibition in vivo. J Biol Chem 2006;281:28993-29001. [PubMed: 16870613]

16. Garrett TPJ, et al. Crystal structure of a truncated epidermal growth factor receptor extracellular domain bound to transforming growth factor alpha. Cell 2002;110:763-773. [PubMed: 12297049]

17. Ogiso $\mathrm{H}$, et al. Crystal structure of the complex of human epidermal growth factor and receptor extracellular domains. Cell 2002;110:775-787. [PubMed: 12297050]

18. Lawrence MC, Colman PM. Shape complementarity at protein/protein interfaces. J Mol Biol 1993;234:946-950. [PubMed: 8263940]

19. Huai Q, et al. Structure of human urokinase plasminogen activator in complex with its receptor. Science 2006;311:656-659. [PubMed: 16456079]

20. Rösel M, Claas C, Seiter S, Herlevsen M, Zöller M. Cloning and functional characterization of a new phosphatidyl-inositol anchored molecule of a metastasizing rat pancreatic tumor. Oncogene 1998;17:1989-2002. [PubMed: 9788443]

21. Temerinac S, et al. Cloning of PRV-1, a novel member of the uPAR receptor superfamily, which is overexpressed in polycythemia rubra vera. Blood 2000;95:2569-2576. [PubMed: 10753836]

22. Hansen LV, Laerum OD, Illemann M, Nielsen BS, Ploug M. Altered expression of the urokinase receptor homologue, $\mathrm{C} 4.4 \mathrm{~A}$, in invasive areas of human esophageal squamous cell carcinoma. Int $\mathrm{J}$ Cancer 2008;122:734-741. [PubMed: 17849475]

23. Kenny PA, Bissell MJ. Targeting TACE-dependent EGFR ligand shedding in breast cancer. J Clin Invest 2007;117:337-345. [PubMed: 17218988]

24. Zhou BB, et al. Targeting ADAM-mediated ligand cleavage to inhibit HER3 and EGFR pathways in non-small cell lung cancer. Cancer Cell 2006;10:39-50. [PubMed: 16843264]

25. Fujimoto N, et al. High expression of ErbB family members and their ligands in lung adenocarcinomas that are sensitive to inhibition of epidermal growth factor receptor. Cancer Res 2005;65:1147811485. [PubMed: 16357156]

26. Hynes NE, Schlange T. Targeting ADAMS and ERBBs in lung cancer. Cancer Cell 2006;10:7-11. [PubMed: 16843261]

27. Borrell-Pagès M, Rojo F, Albanell J, Baselga J, Arribas J. TACE is required for the activation of the EGFR by TGF-alpha in tumors. EMBO J 2003;22:1114-1124. [PubMed: 12606576]

28. Emsley P, Cowtan K. Coot: model-building tools for molecular graphics. Acta Crystallogr D Biol Crystallogr 2004;60:2126-2132. [PubMed: 15572765]

29. CCP4 (Collaborative Computational Project Number 4). The CCP4 suite: Programs for protein crystallography. Acta Crystallogr D Biol Crystallogr 1994;50:760-763. [PubMed: 15299374]

30. Lu HS, et al. Crystal structure of human epidermal growth factor and its dimerization. J Biol Chem 2001;276:34913-34917. [PubMed: 11438527] 
a

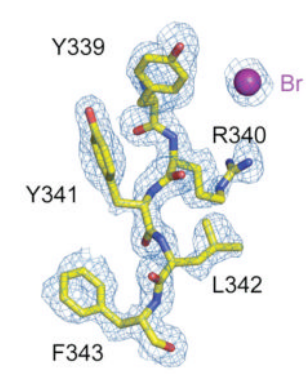

b

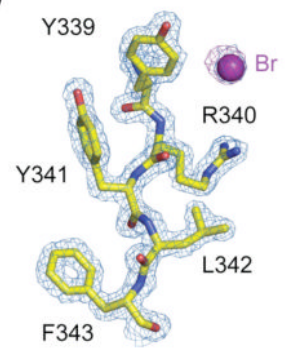

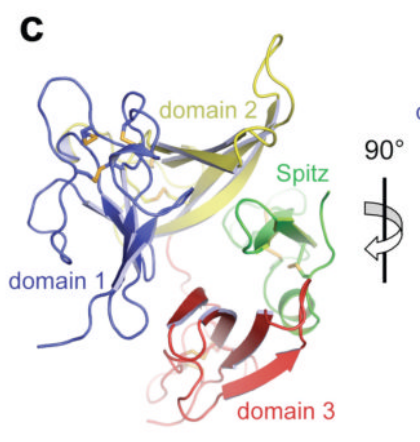
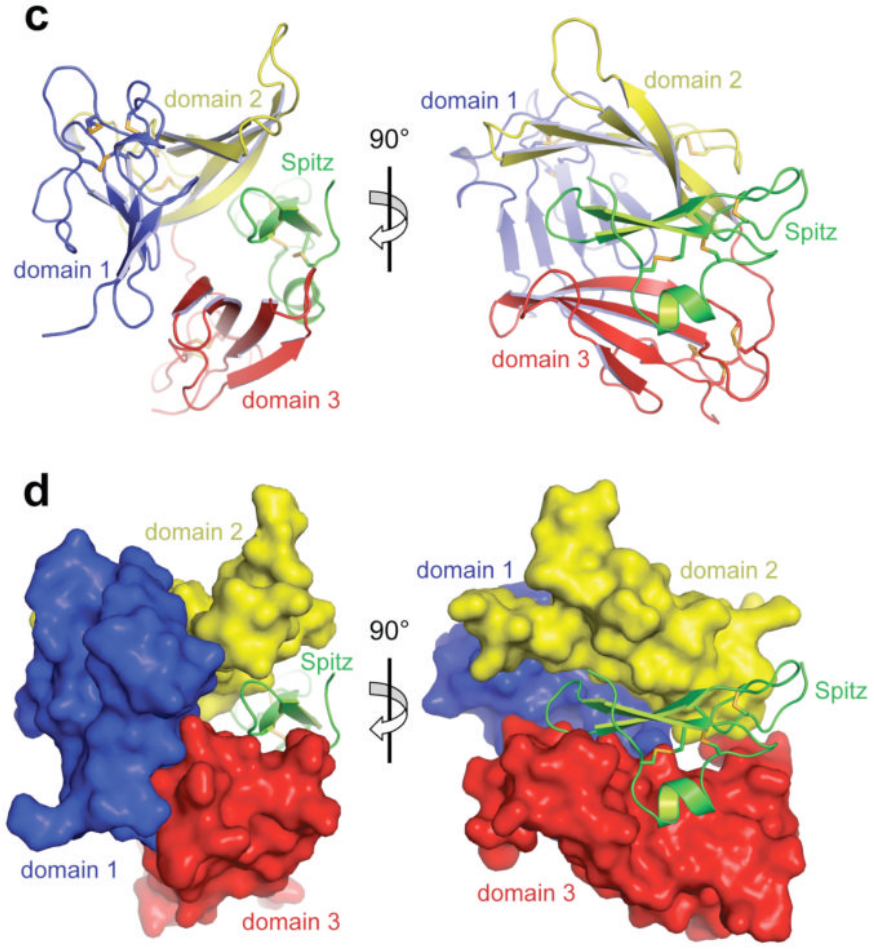

e

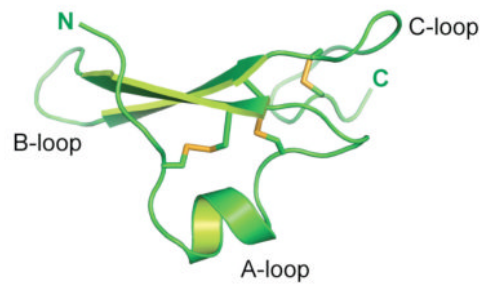

Figure 1. Structure of the Argos-Spitz complex

a, Representative experimental electron density (contoured at $1 \sigma$ ) obtained after MAD phasing, showing a region of the Spitz-binding site on domain 2 (d2A: see Fig 3). The initial model is shown placed in the density. b. The same region of an $2 \mathrm{Fo}-\mathrm{Fc}$ map (contoured at $2 \sigma$ ) calculated using final phases. The final model is shown placed in the density. In purple, a peak corresponding to a bromide ion is seen in the anomalous difference Fourier map (contoured at $4 \sigma$ ) using Br peak data. c, Cartoon of the Argos $217:$ Spitz $_{\text {EGF }}$ complex. Domains 1, 2 and 3 are coloured blue, yellow and red respectively. Spitz is green. Disulphide bridges are coloured orange. Two orthogonal views are shown. d, Same as in c, but with Argos in surface 
representation. e, Cartoon representation of the Spitz EGF domain structure, with A-, B-, and C-loops marked. 
a

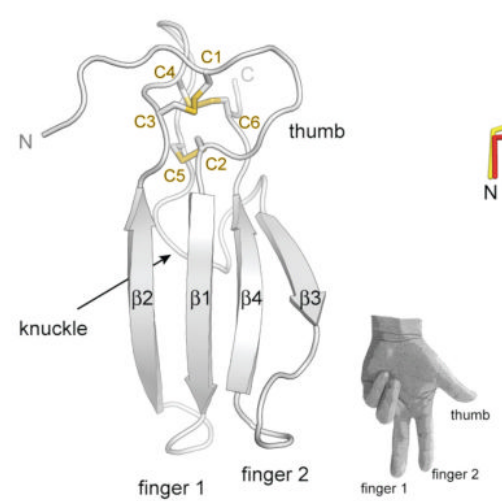

b

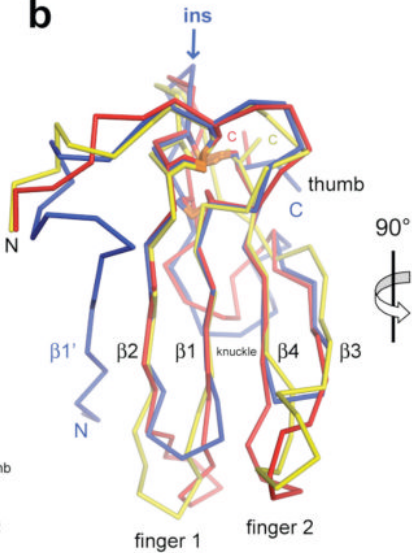

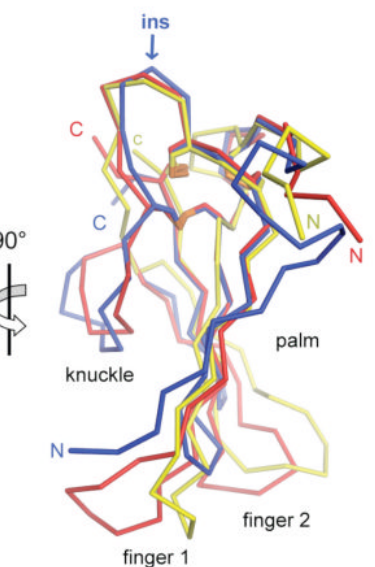

C

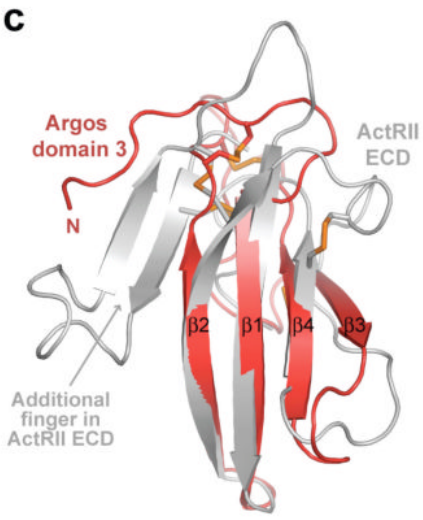

Figure 2. Argos has three similar domains that resemble the 3-finger toxin fold of TGF $\beta$ receptors a, The overall fold of the three constituent domains in Argos is illustrated using domain 3 . The four strands ( $\beta 1-\beta 4$ ) form two fingers (fingers 1 and 2 ) that resemble those on a left hand (as shown). The 'palm' side of the domain faces out of the page. A knuckle-like protrusion projects below the page. At the top of the domain is a disulphide-bonded core from which emanate the two fingers plus the thumb (marked). Cysteines $\mathrm{C} 1$ to C6, which make C1-C3, C2-C5 and C4C6 disulphides are labelled, as are $\mathrm{N}$ - and C-termini. b, Domains 1, 2, and 3 are overlaid (as $\mathrm{C} \alpha$ ribbons) in the same orientation used in a. Colours are as in Figure 1. Strand $\beta 1^{\prime}$, unique to domain 1 is labelled, as is the location of the 120 residue insert (ins) removed to generate $\operatorname{Argos}_{217}$. Two orthogonal views are shown. c, Domain 3 of Argos (red) is overlaid with the 100 aa extracellular ligand-binding domain of the type II activin receptor receptor (ActRII) ${ }^{14}$ (coloured light grey: from pdb entry 2GOO). 


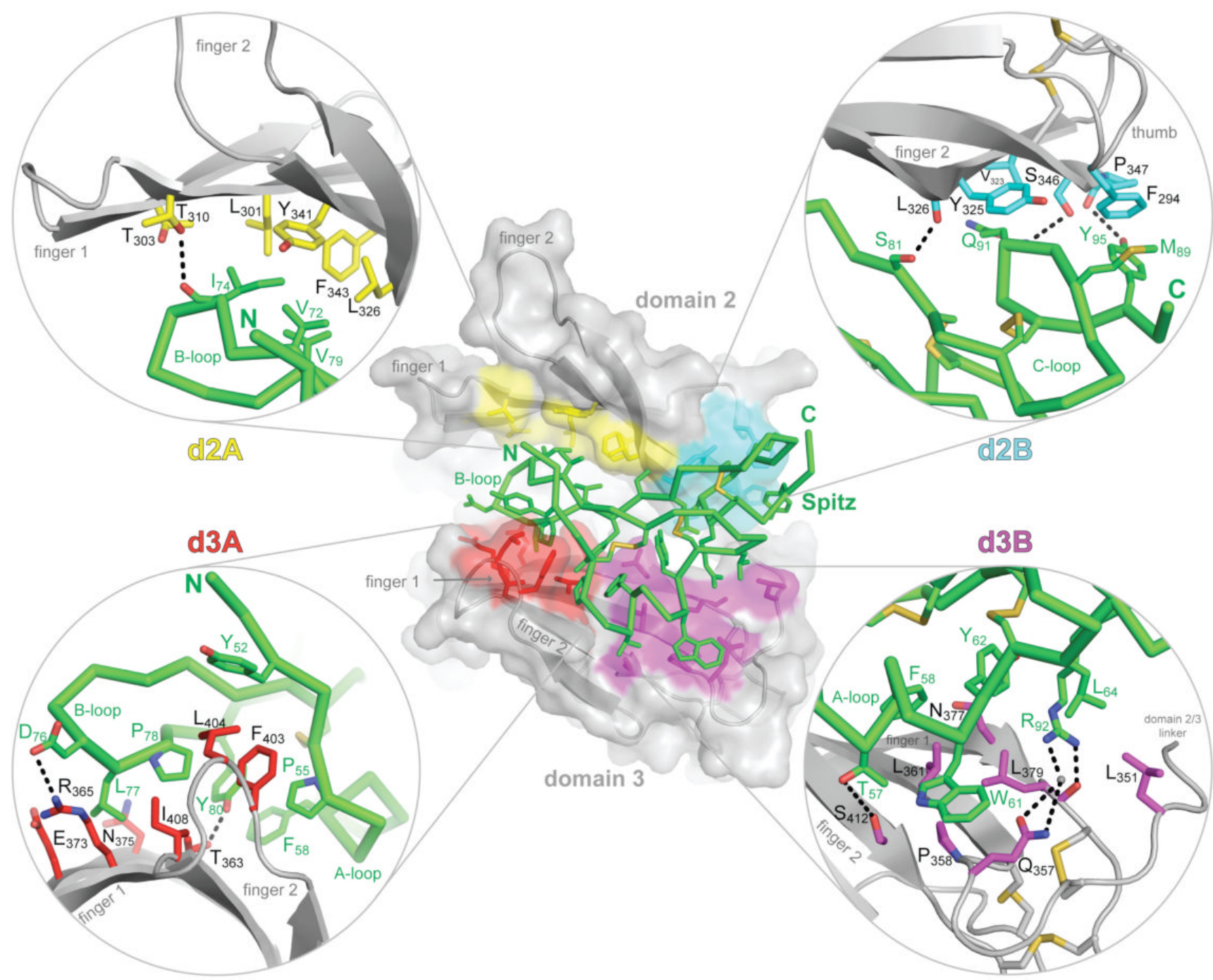

Figure 3. Spitz-binding interactions

The centre panel shows the Argos 217:Spitz EGF complex in an orientation similar to that shown in Fig. 1d (right-hand panel) with Argos coloured grey, and Spitz green. Domains 2 and 3 are marked, as are their two fingers (which project to the left). Four individual Spitz-binding subsites are identified: d2A (yellow), d2B (cyan), d3A (red), and d3B (magenta). Surfaces of side-chains involved in each subsite are coloured accordingly. In each of the four corners, details of an individual subsite are shown, with Argos side-chains coloured for the site. Spitz is green in all panels, and the Argos backbone is grey. 


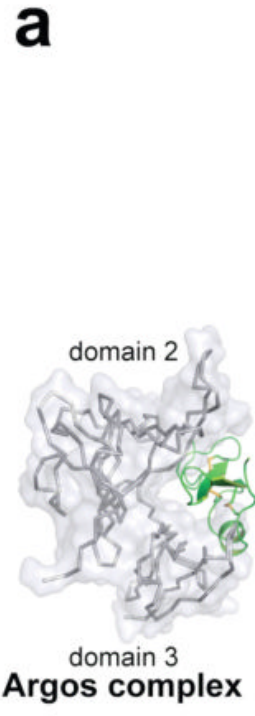

Argos domain 2
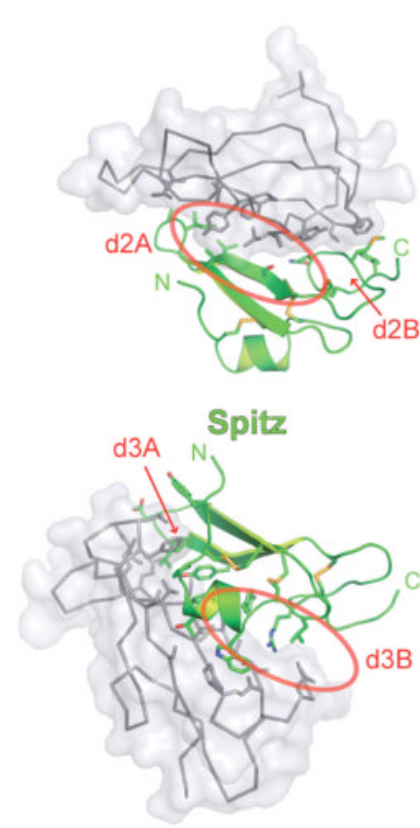

Argos domain 3

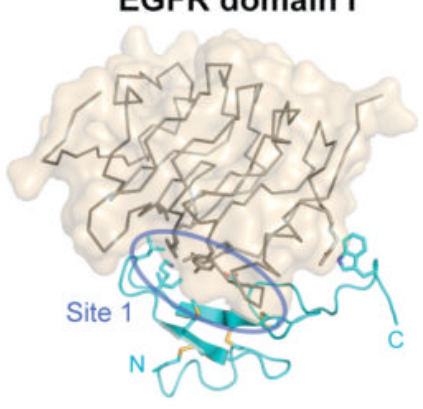

hEGF

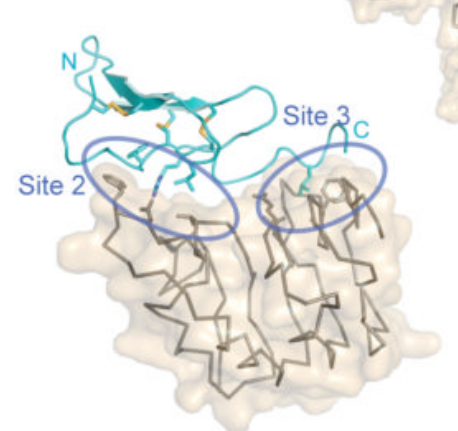

EGFR domain III

\section{b}
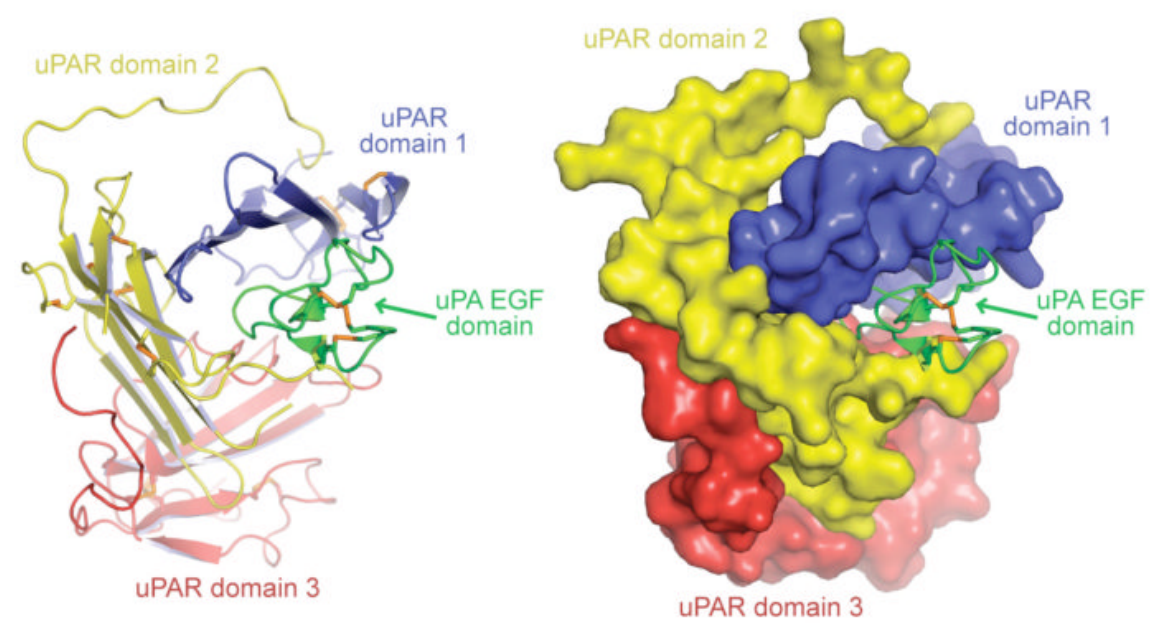

Figure 4. Argos, EGFR, and structural homologues entrap the EGF domain with two binding sites a, The left-most and right-most panels show EGF domains bound to Argos and the human EGFR extracellular region ${ }^{17}$ (sEGFR) respectively. Spitz is green and hEGF cyan. In the central upper panels, Spitz $\mathrm{EGF}_{\text {and }}$ hEGF are shown (in identical orientations) bound to Argos domain 2 (grey) and sEGFR domain I (beige). The side-chains of EGF domain-interacting residues are drawn. Site 1 on sEGFR domain I (defined by Ogiso et al. ${ }^{17}$ ) and its counterpart on Argos (which includes site d2A) are marked by blue and red ovals respectively. In the lower central panels, Spitz $\mathrm{EGF}_{\text {and }}$ hEGF (again in identical orientations) are shown bound to Argos domain 3 and sEGFR domain III. Sites 2 and 3 in the sEGFR/hEGF interface are marked with blue ovals. Argos site d3B mimics sEGFR site 2, but Argos does not mimic sEGFR site 3. 
Instead, Argos makes a unique set of interactions with $\operatorname{Spitz}_{\mathrm{EGF}}$ (site d3A). A key aliphatic side-chain critical for hEGF binding to site 3 of EGFR (L47 in hEGF, I98 in Spitz) is disordered and exposed in the Spitz $\mathrm{EGF}_{\text {A }}$ Argos complex. b, Domain organization of the uPA receptor ${ }^{9}$, 19. The three domains in UPAR are coloured with the order used for Argos in Fig. 1. Like Argos, uPAR uses three copies of this domain type - although in a different arrangement - to form a C-clamp-like structure for enveloping an EGF domain 9,19 . 